\title{
An emission model tracking the life cycle pathways of human pharmaceuticals in Korea
}

\author{
Eun Jeong Han $\cdot$ Hee Seok Kim $\cdot$ Dong Soo Lee
}

Received: 27 June 2013/Accepted: 9 July 2013/Published online: 11 August 2013

(C) The Japanese Society for Hygiene 2013

\begin{abstract}
Objectives Pharmaceuticals in the environment are of growing public health concern. The main objectives of this study were to develop a new emission estimation model, identify factors critical to reducing emission, and demonstrate the model's applicability for screening and priority setting.

Methods A new emission estimation model was developed covering the life cycle pathways of pharmaceuticals from supply to discharge into surface water. The emission estimates of the model were assessed by coupling with SimpleBox to give predicted concentrations and by comparing the predicted concentrations with measured concentrations in Korean surface waters for five selected pharmaceuticals (acetaminophen, cephradine, ibuprofen, mefenamic acid, and naproxen).

Results The sensitivity analysis revealed that the biodegradation rate in the sewage treatment plant and the excretion rate of pharmaceuticals were the most important factors influencing the emission rate. The uncertainty of the emission estimate was found to increase with increases in
\end{abstract}

Electronic supplementary material The online version of this article (doi:10.1007/s12199-013-0352-8) contains supplementary material, which is available to authorized users.

\author{
E. J. Han · H. S. Kim · D. S. Lee $(\bowtie)$ \\ Environmental Planning Institute, Graduate School \\ of Environmental Studies, Seoul National University, \\ Gwanak-ro 1, Gwanak-ku, Seoul 151-742, \\ Republic of Korea \\ e-mail: leeds@snu.ac.kr \\ E. J. Han \\ e-mail: hikate05@snu.ac.kr \\ H. S. Kim \\ e-mail: dawit75@snu.ac.kr
}

the value of the emission estimate. Once the intrinsic properties of a pharmaceutical (excretion rate, biodegradation rate, and removal rate by sludge separation) were given, the patient behavior parameters, such as participation in a Take-back program and rate of administration, were determined to have a strong influence on the emission estimate. In our study, the predicted and measured concentrations agreed with each other within one order of magnitude. Several management implications were drawn from the analysis of model outcomes.

Conclusions The model outcomes, alone or in combination with toxicity data, may potentially be used for the purposes of screening, priority setting, and the design of management programs.

Keywords Pharmaceuticals - Emission model · Pathway $\cdot$ Uncertainty $\cdot$ Sensitivity

\section{Introduction}

The presence of pharmaceuticals in aquatic environments was initially determined in the late 1990s [1-3], and since this time concerns regarding their presence in the environment among public and scientific communities have been rapidly increasing [4]. Increasing evidence of the potential ecological risks posed by the levels of certain pharmaceuticals that have often been found in aquatic environments [5] testifies to the need to develop management options prior to or following the discharge of these pharmaceuticals to minimize adverse health effects. To develop such management programs, a knowledge of emission rates of pharmaceuticals is essential.

The emission rate can be determined either by direct measurement or by using estimation methods. Direct 
measurement of numerous pharmaceuticals, however, may be neither practical nor applicable considering the myriads of pharmaceutical compounds being used and also the difficulties in performing analytical measurements. In fact, the quantity and quality of existing environmental occurrence data on pharmaceuticals are insufficient for decisionmaking [5]. In Korea, only a limited number of studies have been published on the occurrence of pharmaceuticals in surface water [6-13] or in sewage treatment plant (STP) effluents [7, 10,14]. While providing valuable information, these studies are fragmentary in nature and do not provide sufficient data for estimating an emission rate at either the national or local level.

This lack of data has led to the development of various emission estimation methods which do not require measurement data. One of the simplest estimation models is that of U.S. Food and Drug Administration (FDA) which employs a formula to calculate the expected introduction concentration (EIC) of pharmaceuticals. Using a dilution factor of 10, one can estimate the predicted environmental concentration (PEC) from the EIC [15]. The FDA method is based on the very conservative assumption that $100 \%$ of each individual pharmaceutical that is produced is consumed and enters the publicly owned treatment works system. The European Agency for the Evaluation of Medical Products (EMEA) of the European Union has also developed a formula to estimate PEC [16]. While the EMEA method appears to be more realistic than the FDA method in that important fate processes, such as the excretion rate and STPs, are factored in for the emission estimation, this approach also has room for improvement. For example, this method does not consider the factors affecting the emission rate in the life cycle stages of pharmaceuticals, including distribution in the supply chain, consumption, disposal, and waste treatment of pharmaceuticals. Specifically, the quantity of disuse and treatment efficiency of the disuse step in the supply chain (pharmaceutical companies, importers, wholesalers, hospitals, and pharmacies) can significantly alter the emission rate. The excretion rate from the human body and the biodegradation rate in STPs varies depending on the properties of individual pharmaceuticals, thereby directly influencing their emission rates. Consequently, taking these factors into account in the emission estimation process will improve the accuracy of the estimate. In addition, the consideration of life cycle factors can provide information on the contribution of individual stages or factors to the overall emission rate, which is essential to the development of an efficient emission reduction strategy. Although the need for considering the life cycle of pharmaceuticals in emission estimation has been suggested [17-19], it still remains to be realized because the scientific data on their source, fate, and transport are limited and uncertain [5]. Consideration of important factors in the life cycle of pharmaceuticals is therefore an important challenge in developing emission models of improved accuracy.

The main objectives of our study were to (1) develop a new emission estimation model, (2) identify factors critical to reducing emission, and (3) demonstrate the model's applicability for screening and priority setting.

\section{Methods}

Emission estimation model development

For model development, we first constructed a framework of life cycle of pharmaceuticals in Korea by tracking all of the pathways from distribution following production and/or import to final discharge into surface water. Although the life cycle in this present study is incomplete due to exclusion of the production and import stages, the uncertainty of the exclusion was assumed to be negligible relative to the total emission rate. After the construction of the life cycle framework, a set of equations was formulated for calculating the amounts of pharmaceuticals involved in the pathways and stages of the life cycle using the parameters and variables identified to be necessary for the calculation.

\section{Model assessment}

To assess the accuracy of the emission estimates, the PEC was calculated by using the emission estimates and compared with the measured environmental concentration (MEC) available for surface waters in Korea [20].

A modified version of SimpleBox (ver. 3.24a) was used to calculate the PEC from the emission estimates. The modification systematically included two aspects. First, the transport of chemicals between the regional compartment and the continental/global compartment was nullified because it is not a relevant factor for surface water quality in Korea, especially with chemicals of low vapor pressure. Second, many parameter values given in the original SimpleBox were replaced with those representing Korea's environmental and meteorological settings. A modified version of SimpleTreat (ver. 3.1) was used to calculate the biodegradation rate, removal rate by sludge separation, and volatilization loss rate depending on the chemical properties of the selected pharmaceuticals and average operation conditions of STPs in Korea.

For assessing the accuracy of the model estimates, we selected five target pharmaceuticals (acetaminophen, cephradine, ibuprofen, mefenamic acid, and naproxen) because (1) their MECs [20] were available to compare with the PECs in our study, (2) they were considered to have high management priority in Korea [11-13], and (3) 
they were primarily used for human consumption. Details of these pharmaceuticals are presented in Electronic Supplementary Material (ESM) 1. The total production volume in 2009 was calculated from the production data [21] and information on the active ingredient(s) in each medicinal product $[22,23]$. The excretion rate was obtained from the American Society of Health-System Pharmacist's DI [24], and the biodegradation rate in STPs and the removal rate by sludge separation in STPs were calculated by the modified SimpleTreat.

\section{Uncertainty and sensitivity analysis}

For the uncertainty assessment, Monte-Carlo calculations were conducted by using Crystal Ball ${ }^{\circledR}$ (ver. 11.1.1.1.00; Oracle Corp., Redwood, CA). As no prior information was available on the distribution shape of the parameters/variables used in the model, a uniform distribution was assigned to each of parameters/variables. We performed 100,000 trials for each Monte-Carlo run and recorded five statistics (minimum, maximum, range, median, and skewness) to assess the uncertainty of the model estimate of the emission.

To identify sensitive parameters/variables that strongly affect the model estimates, we used the rank correlation coefficient normalized to $100 \%$ [25] as an index of sensitivity. The rank correlation coefficient was provided in the sensitivity analysis function of Crystal Ball ${ }^{\circledR}$.

\section{Results}

Emission estimation model

The pathways of human pharmaceuticals in the model are depicted in Fig. 1. Pharmaceuticals produced or imported are primarily supplied to domestic buyers, including pharmaceutical companies, pharmacies, hospitals, wholesalers, and others, and finally to patients through hospitals and pharmacies which are the exclusive official pathways to patients in Korea. All disuse from wholesalers, hospitals, and pharmacies is principally incinerated. A pharmaceutical supplied to a patient would be excreted following administration or, if not administered, wasted or taken back [Take-back program (TBP)]. A certain fraction of the administered pharmaceutical is excreted (ER) into a toilet connected with a septic tank (SEPT) and subsequently transported to the nightsoil treatment plant (NISO). All of the pharmaceuticals collected through the TBP are incinerated $[26,27]$. Of the wasted pharmaceuticals, the portion disposed of in the toilet is sent to NISO, the portion disposed of in the sink enters the sewer to reach the STP, and the portion disposed of in the waste bin eventually reaches the landfill leachate treatment plant (LEACH). The residue after incineration of the disused or taken-back pharmaceuticals undergoes treatment by STP or LEACH. It is to be noted, therefore, that all of the marketed human pharmaceuticals in Korea are subject to one or more treatment processes before entering surface waters (Fig. 1).

A total of 100 parameters/variables are used in the model (ESM 2). The important parameters/variables summarized in Table 1 are divided into three groups, i.e., (1) variables for which the value is known or can be estimated for individual pharmaceuticals, (2) parameters for which a single fixed value $[28,29]$ was used commonly for all pharmaceuticals, and (3) parameters for which the range was assumed due to the lack of sufficient information [30]. A single value was assigned to the supply rates (SR) in Table 1 because the value is not expected to vary considerably among pharmaceuticals.

In our study, we made four assumptions regarding the parameters/variables in Table 1 and ESM 2. First, the removal rate by sludge separation in LEACH and NISO, for which values were unavailable, were assumed to be the same as those in the STP (SLR.stp) because the sludge removal processes are often similar. Likewise, the biodegradation rate in LEACH was assumed to be the same as that in STP (BR.stp). Second, the biodegradation in NISO was assumed to be negligible. Most NISOs in Korea are designed to perform preliminary treatments, such as solid separation, and are connected to STPs for further treatment. Third, the removal by incineration (INCN) was assumed to be complete. Due to the public concern for dioxins in Korea, the incineration temperature is required to be maintained above $850{ }^{\circ} \mathrm{C}$, at which temperature pharmaceuticals would be completely destroyed. Consequently, as the removal by INCN is assumed to be complete, the landfill rate of incineration residue (LFR.incn) becomes zero in our study. Finally, although the return rate to the Take-back program (TBR) appeared to vary annually, the ratio among the three waste rates [waste bin (WR.wb), sink (WR.sink), and toilet (WR.toilet)] were assumed to be constant at 86:7:7 as found in the survey of 2009 [26]. By using the inputs and assumptions described above, we identified a total of 57 model outputs, as summarized in ESM 2.

\section{Model assessment}

As shown in Fig. 2, the PECs calculated using the emission estimates of the model were compared with the MECs [20]. The median and range of PECs were obtained from using those of the emission rates estimated by the model and adjusted by the modified SimpleTreat for removal efficiency, respectively, as inputs to the modified SimpleBox. Figure 2 shows that the PECs of the selected pharmaceuticals agreed with the MECs for the median within one order of magnitude. 
Fig. 1 Schematic of the pharmaceutical emission estimation model in the present study. See ESM 2 for definition of parameters/variables in the scheme

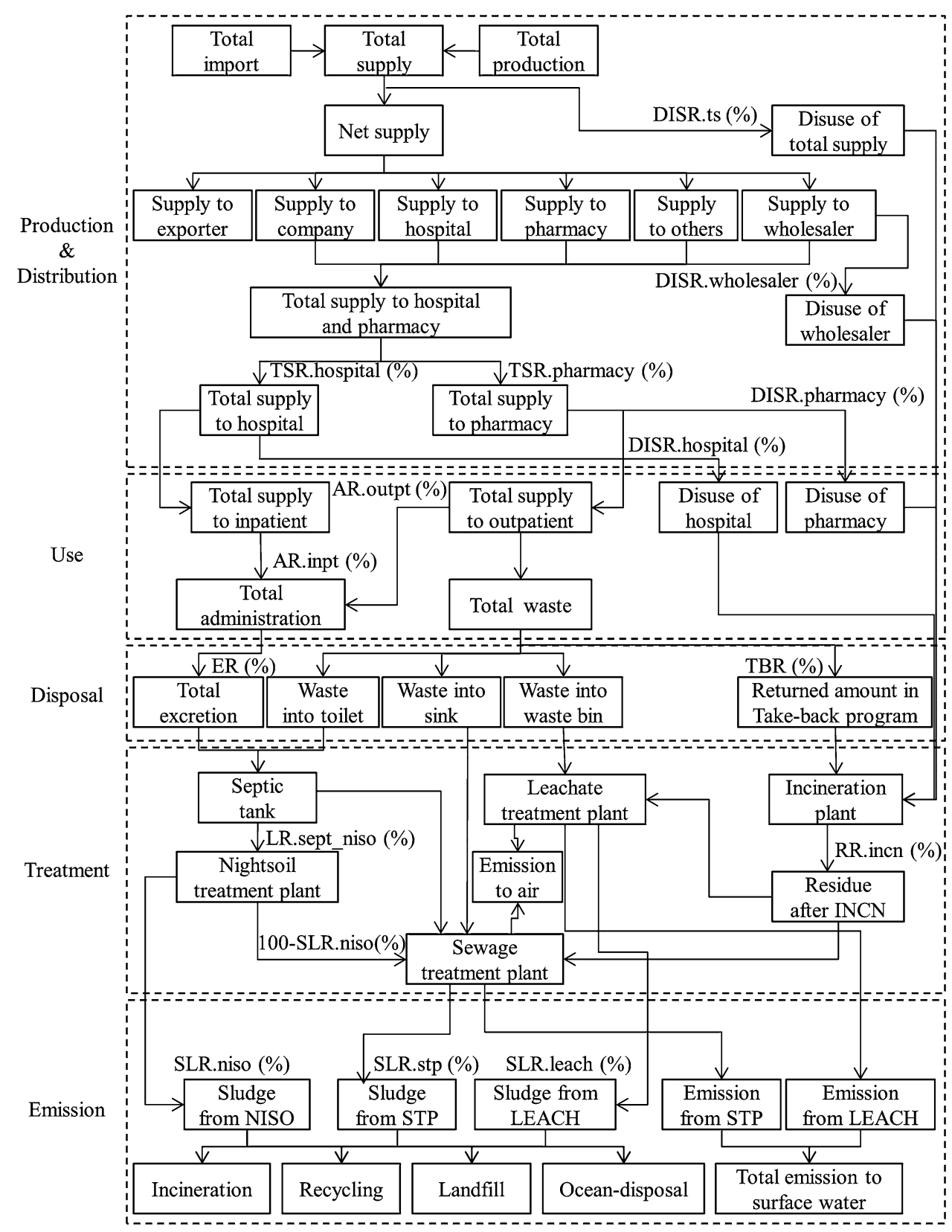

Mass flow along the pathways of pharmaceuticals

The emission estimation model can be used to estimate the amounts of pharmaceuticals in various steps along the pathways as well as the final emission into surface water. For the model application, 14 pharmaceuticals were selected in addition to those shown in Fig. 2. These pharmaceuticals also meet the priority criteria applied in our study to assess the model accuracy except that they are also used extensively for veterinary purposes. The mass flows of the 19 selected pharmaceuticals are summarized in Table 2. The value in each step is the median of predicted distribution by Monte-Carlo runs of 10,000 repetitions with the sum of production and import (TS) of 100. The median of TE.water was found to range from 0.6 to $40.3 \%$ of the TS, with the medians for roxithromycin, trimethoprim, ciprofloxacin, cephradine, and cefadroxil having the five highest values $(>20 \%)$.

Risk characterization and priority setting

Using the emission estimation model enabled the risk characterization to be performed in combination with toxicity data. For example, hazard quotients (HQ) were calculated for the 19 pharmaceuticals used in the model application, as shown in Fig. 3. All of the HQs of these 19 
Table 1 Model parameters

\begin{tabular}{|c|c|c|}
\hline Name & Description & Value or equation \\
\hline AR.inpt & Administration rate of inpatient $(\%)$ & $100^{\mathrm{a}}$ \\
\hline AR.outpt & Administration rate of outpatient $(\%)$ & $39.1-100.0^{\mathrm{b}}$ \\
\hline BR.stp & Biodegradation rate in sewage treatment plant $(\%)$ & Pharmaceutical specific value \\
\hline DISR.hospital & Disuse inventory ratio in hospitals $(\%)$ & $0.0-10.0^{\mathrm{c}}$ \\
\hline DISR.pharmacy & Disuse inventory ratio in pharmacies $(\%)$ & $0.0-10.0^{\mathrm{c}}$ \\
\hline DISR.ts & Disuse inventory ratio in total supplies (\%) & $0.0-10.0^{\mathrm{c}}$ \\
\hline DISR.wholesaler & Disuse inventory ratio in wholesalers $(\%)$ & $0.0-10.0^{\mathrm{c}}$ \\
\hline ER & Excretion rate $(\%)$ & Pharmaceutical specific value \\
\hline INCN.in & Inflow of pharmaceuticals to incineration plant (kg/year) & DIS.ts + DIS.w + DIS.h + DIS.p + TB \\
\hline LEACH.in & Inflow of pharmaceuticals to leachate treatment plant (kg/year) & W.wb + RE.incn·LFR.incn/100 \\
\hline LFR.incn & Landfill rate out of incineration residue (\%) & $0.0^{\mathrm{d}}$ \\
\hline LR.sept_niso & Linked treatment rate of septic tank to nighsoil treatment plant (\%) & $73.6^{\mathrm{e}}$ \\
\hline NISO.in & Inflow of pharmaceuticals to nightsoil treatment plant (kg/year) & SEPT.in·LR.sept_niso/100 \\
\hline NS & Net supply (kg/year) & TS-DIS.ts \\
\hline RR.incn & Removal rate in incineration plant $(\%)$ & $100^{\mathrm{f}}$ \\
\hline SEPT.in & Inflow of pharmaceuticals to septic tank ( $\mathrm{kg} / \mathrm{year})$ & TA.excretion + W.toilet \\
\hline SL.tot & Total residue in sludge from water treatment plants (kg/year) & SL.stp + SL.leach + SL.niso \\
\hline SLR.stp & Removal rate by sludge separation in sewage treatment plant (\%) & Pharmaceutical specific value \\
\hline SR.hospital & Supply rate to hospital (\%) & $25.5^{\mathrm{g}}$ \\
\hline SR.pharmacy & Supply rate to pharmacy $(\%)$ & $18.1^{\mathrm{g}}$ \\
\hline STP.in & Inflow of pharmaceuticals to sewage treatment plant (kg/year) & W.sink + L.setp_stp + L.niso_stp + L.incn_stp \\
\hline TA.imp & Total amount of import (kg/year) & Pharmaceutical specific value \\
\hline TA.prod & Total amount of production (kg/year) & Pharmaceutical specific value \\
\hline TB & Returned amount in Take-back program (kg/year) & TA.waste·TBR/100 \\
\hline TBR & Return rate to Take-back program (\%) & $0-100$ \\
\hline TE.water & Total emission to surface water (kg/year) & E.stp + E.leach \\
\hline TS & Total supply to region (kg/year) & TA.prod + TA.imp \\
\hline WR.sink & Waste rate into sink (\%) & $(100-\mathrm{TBR}) \cdot 0.07^{\mathrm{h}}$ \\
\hline WR.toilet & Waste rate into toilet (\%) & $(100-\mathrm{TBR}) \cdot 0.07^{\mathrm{h}}$ \\
\hline WR.wb & Waste rate into waste bin (\%) & $(100-\mathrm{TBR}) \cdot 0.86^{\mathrm{h}}$ \\
\hline
\end{tabular}

${ }^{a}$ Inpatients are hardly allowed to waste prescribed medications

b The minimum value of $39.1 \%$ for AR.outpt was taken from the rate of people who take all the medications they purchase in a survey performed in 2009 [26]

c According to a survey performed in 2009 [30], these rates were reported to be $<10 \%$

d Assumed to be negligible

e 2009 data from Sewerage of Korea statistics [29]

f Assumed to be complete

g 2009 data from Korean pharmaceutical industry statistics [28]

h The ratio of 86:7:7 was taken from a survey in 2009 [26]

pharmaceuticals were found to be smaller than unity even with the maximum PECs, indicating that each of the pharmaceuticals may not pose significant ecological risk. Nonetheless, precaution needs to be taken against the potential combined effects [31], particularly for those pharmaceuticals with a HQ value near one. The ranks by HQ, PEC, toxicity, and emission of the 19 selected pharmaceuticals are summarized in Table 3.

\section{Discussion}

Factors critical to emission estimates of pharmaceuticals

Due to a lack of information we were unable to assign a single value to each of six input parameters (administration rate of outpatients, return rate to Take-back program, 
disuse inventory ratio in total supplies/wholesalers/hospitals/pharmacies). Therefore, the influence of uncertainties associated with these six parameters needs to be assessed on the emission estimates of the model. Moreover, for the assessment of uncertainty of the model estimate when no specific pharmaceutical is specified (termed "general uncertainty" hereafter), the influence of the variability of two pharmaceutical-dependent variables (ER and BR.stp,

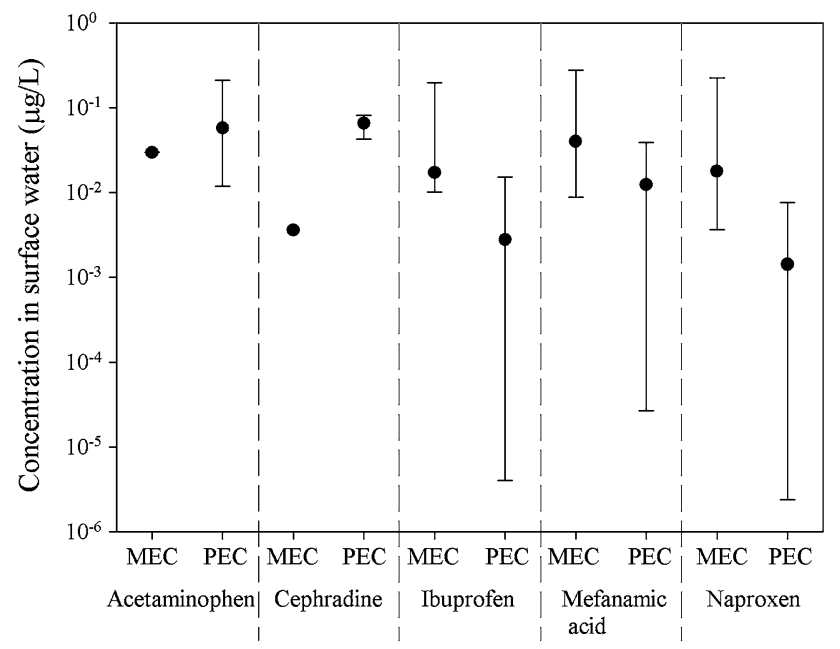

Fig. 2 Comparison of predicted environmental concentration (PEC) with the measured environmental concentration $(M E C)$ for selected pharmaceuticals. Filled circles Mean for MEC and median for PEC, whiskers range
SLR.stp) should also be assessed. An arbitrary value of 100 for the sum of production and import (TS) was assigned to assess the general uncertainty of the model estimate of the emission.

As shown in Fig. 4a, the general uncertainty of the model estimate for emission (TE.water) could vary from 0.0 to $83.0 \%$ (median value $15.0 \%$ ) of TS. The distribution is positively skewed, i.e., half of the TE.water values are below $17.2 \%$ of the range. The uncertainty of this magnitude strongly suggests a need to acquire accurate values for the uncertain parameters/variables, particularly for those of high sensitivity. Based on the magnitude of the rank correlation coefficients, the two most sensitive parameters/variables were identified to be ER and BR.stp, with a large gap between these and the following parameter, TBR, as shown in Fig. 4b. The impacts of the remaining parameters/variables were negligible.

To investigate further the influence of BR.stp and ER on TE.water, we calculated a probability distribution of TE.water using the Monte-Carlo technique for each of nine $(3 \times 3)$ combinations of BR.stp and ER values of 10, 50, and $90 \%$, respectively. As shown in Fig. 5a, the nine distributions appear to differ substantially in their median and range. For example, under conditions where ER is $90 \%$ and BR.stp is $10 \%$, the median and variation are about 98 -fold greater and 12-fold wider, respectively, than those in the case where ER is $10 \%$ and BR.stp is $90 \%$. This comparison clearly demonstrates the strong influence

Table 2 Percentage of pharmaceuticals in each pathway calculated with emission model of this study

\begin{tabular}{|c|c|c|c|c|c|}
\hline Pharmaceuticals & INCN.in & LEACH.in & NISO.in & STP.in & TE.water \\
\hline Acetaminophen & 16.9 & 4.5 & 3.4 & 5.1 & 1.1 \\
\hline Acetylsalicylic acid & 16.9 & 4.3 & 21.7 & 30.0 & 4.2 \\
\hline Amoxicillin & 16.8 & 4.3 & 32.8 & 45.1 & 15.6 \\
\hline Ampicillin & 16.8 & 4.4 & 21.4 & 29.6 & 10.9 \\
\hline Cefaclor & 17.0 & 4.4 & 36.5 & 50.1 & 17.1 \\
\hline Cefadroxil & 17.0 & 4.5 & 48.0 & 65.8 & 22.0 \\
\hline Cefatrizine & 17.0 & 4.4 & 25.0 & 34.4 & 12.3 \\
\hline Cephradine & 16.9 & 4.6 & 48.0 & 65.7 & 22.1 \\
\hline Cimetidine & 16.8 & 4.4 & 31.0 & 42.4 & 14.7 \\
\hline Ciprofloxacin & 16.9 & 4.4 & 26.5 & 36.6 & 24.2 \\
\hline Diclofenac & 16.8 & 4.4 & 25.2 & 34.0 & 11.8 \\
\hline Erythromycin & 16.9 & 4.3 & 1.6 & 2.7 & 6.8 \\
\hline Ibuprofen & 16.9 & 4.4 & 0.6 & 1.1 & 0.6 \\
\hline Lincomycin & 16.8 & 4.5 & 4.3 & 6.4 & 3.4 \\
\hline Mefenamic acid & 16.9 & 4.6 & 4.9 & 6.8 & 3.4 \\
\hline Naproxen & 17.0 & 4.5 & 0.6 & 1.1 & 0.6 \\
\hline Roxithromycin & 16.9 & 4.5 & 24.8 & 34.3 & 40.3 \\
\hline Streptomycin & 16.7 & 4.4 & 29.6 & 40.7 & 14.3 \\
\hline Trimethoprim & 16.9 & 4.5 & 31.9 & 43.7 & 28.1 \\
\hline
\end{tabular}

Data are given as the percentage of sum of production and import (TS) 
of the two variables on the emission estimate. Furthermore, as shown in Fig. 5b, both the magnitude (as represented by the median of the distribution) and the uncertainty (as represented by the width of the distribution) of TE.water vary in the same direction with ER or BR.stp. For example, the value of TE.water and its uncertainty increase with an increasing ER or decreasing BR.stp. Therefore, greater TE.water will tend to be predicted with a greater

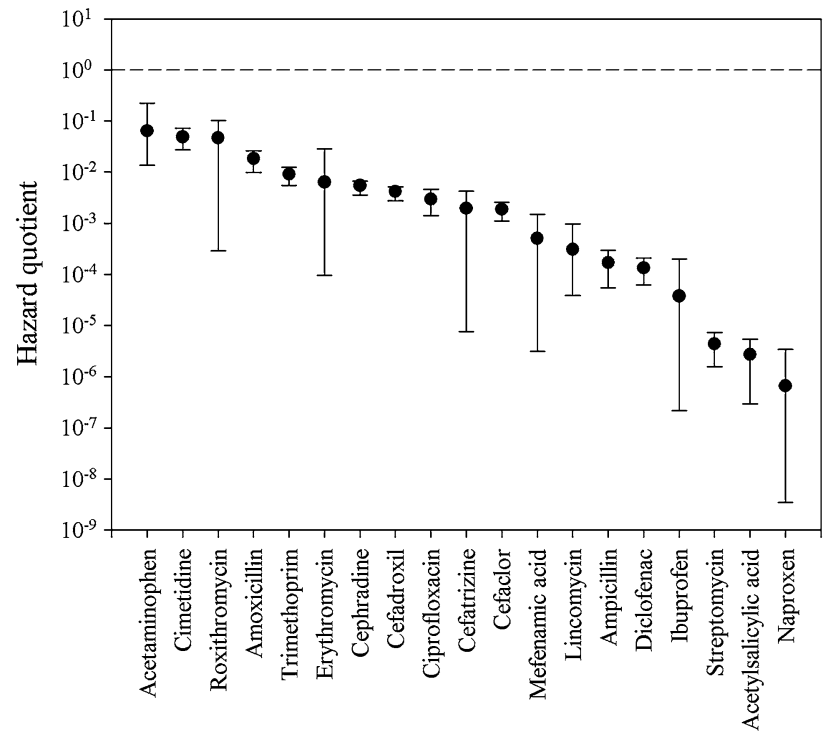

Fig. 3 Hazard quotients of the selected pharmaceuticals uncertainty by the model. It follows that accurate values for ER and BR.stp are particularly critical to the use of the model because (1) they are sensitive variables which could strongly influence the model estimate of emission for any pharmaceutical and (2) without these accurate values, the model estimate would be associated with larger uncertainty, particularly for pharmaceuticals with a higher emission potential (i.e., greater TE.water due to greater ER and/or lower BR.stp).

Once the intrinsic properties of a pharmaceutical (ER, BR.stp, and SLR.stp) are given, patient behavior parameters, such as participation in a Take-back program and administration rate of outpatient (AR.outpt), have strong influence on the emission estimate. When the value of ER and BR.stp is fixed at 90 and $10 \%$, respectively, (i.e., the worst case of emission where TE.water ranges up to $75 \%$ of TS), the uncertainty of TE.water remains fairly constant, as seen in Fig. 6, regardless of the TBR and AR.outpt levels because the uncertainty of TE.water is primarily governed by ER and BR.stp. As shown in Fig. 6, TE.water decreases with TBR more sensitively at lower AR.outpt, obviously suggesting that a consumer Take-back program would have a lower potential for emission reduction for pharmaceuticals with a greater administration rate. Furthermore, the curve of TE.water at AR of $90 \%$ in Fig. 6 indicates that take-back is likely to be of little practical significance for emission reduction when both AR.outpt and ER are high. For these pharmaceuticals, emission
Table 3 Ranking by riskrelated factors for the selected pharmaceuticals

\begin{tabular}{|c|c|c|c|c|}
\hline Pharmaceuticals & $\begin{array}{l}\text { Hazard } \\
\text { quotient }\end{array}$ & $\begin{array}{l}\text { Predicted environmental } \\
\text { concentration }\end{array}$ & Toxicity & $\begin{array}{l}\text { Emission into } \\
\text { surface water }\end{array}$ \\
\hline Acetaminophen & 1 & 8 & 1 & 6 \\
\hline Cimetidine & 2 & 3 & 4 & 2 \\
\hline Roxithromycin & 3 & 1 & 6 & 3 \\
\hline Amoxicillin & 4 & 2 & 7 & 1 \\
\hline Trimethoprim & 5 & 11 & 2 & 13 \\
\hline Erythromycin & 6 & 13 & 3 & 16 \\
\hline Cephradine & 7 & 5 & 9 & 5 \\
\hline Cefadroxil & 8 & 6 & 8 & 7 \\
\hline Ciprofloxacin & 9 & 7 & 10 & 9 \\
\hline Cefatrizine & 10 & 9 & 11 & 8 \\
\hline Cefaclor & 11 & 4 & 15 & 4 \\
\hline Mefenamic acid & 12 & 10 & 12 & 11 \\
\hline Lincomycin & 13 & 17 & 5 & 18 \\
\hline Ampicillin & 14 & 15 & 13 & 14 \\
\hline Diclofenac & 15 & 12 & 17 & 12 \\
\hline Ibuprofen & 16 & 16 & 16 & 15 \\
\hline Streptomycin & 17 & 19 & 14 & 19 \\
\hline Acetylsalicylic acid & 18 & 14 & 19 & 10 \\
\hline Naproxen & 19 & 18 & 18 & 17 \\
\hline
\end{tabular}


Fig. 4 a Predicted distribution of total emissions into surface water, $\mathbf{b}$ sensitivity of the model parameters/variables. STP Sewage treatment plant

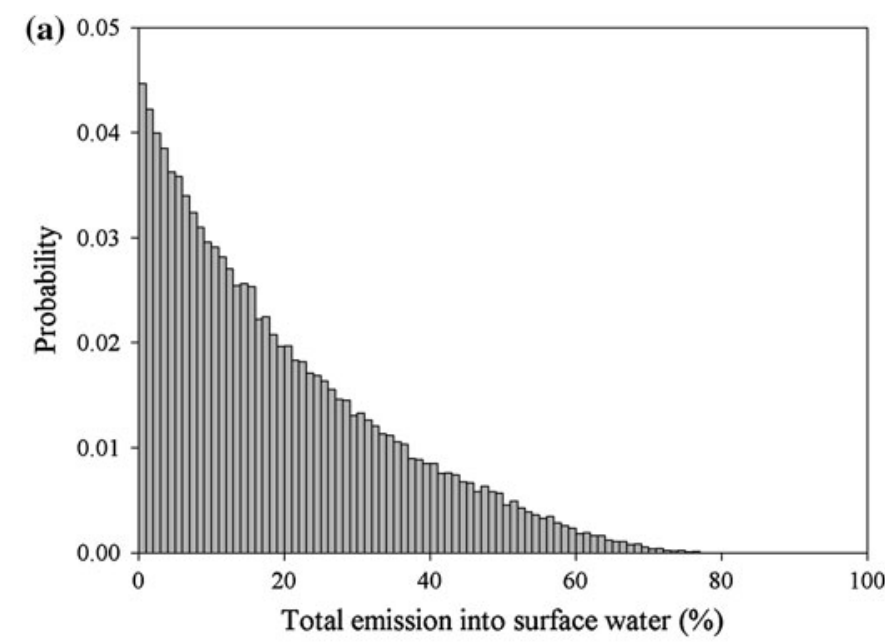

(b)

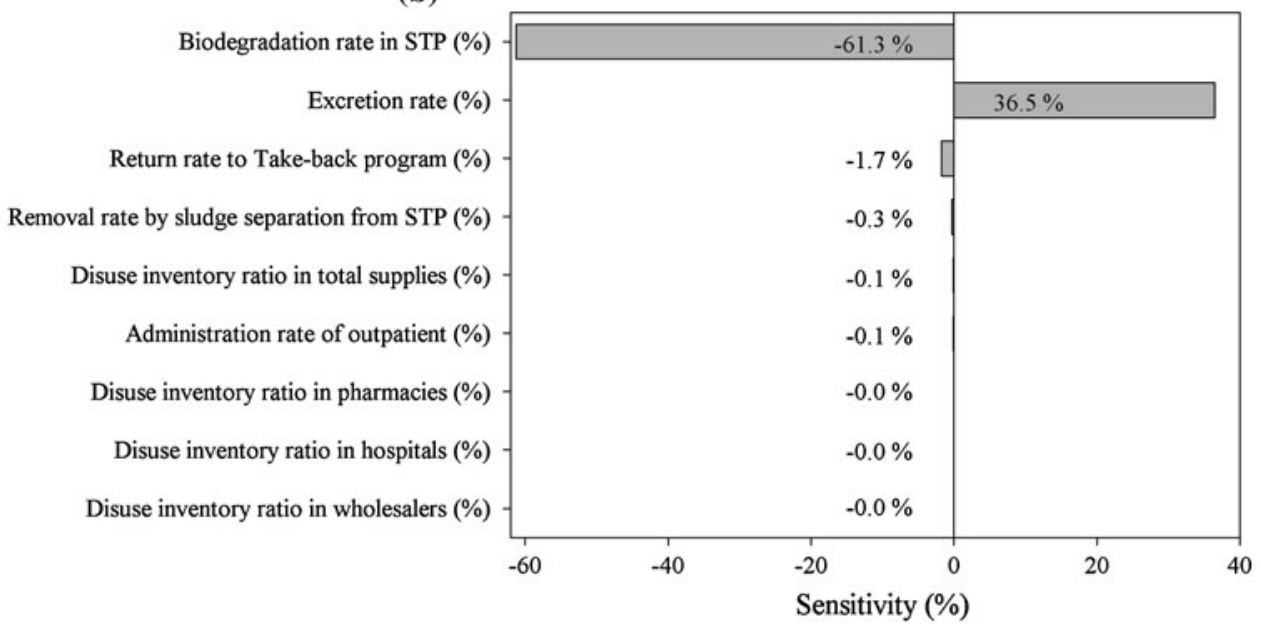

reduction can be theoretically achieved by increasing the removal rate in STP and/or reducing their use. Increasing the removal rate of pharmaceuticals, however, is of secondary concern in STP operation. Therefore, reducing their use appears to be the only viable option within the pathways in Korea.

\section{Model assessment}

The uncertainties in the PECs found in our study (Fig. 2) arise due to (1) the emission estimation model itself and the various data used in the model and (2) the modified SimpleBox and SimpleTreat and their input data. Furthermore, as monitoring data on pharmaceuticals are very limited, it is not certain if the MECs adopted in our study truly represent the contamination levels in surface waters. Taking these sources of uncertainty into account, the emission model that we have developed appears to have a potential to provide reasonable emission estimates for human pharmaceuticals used in Korea.
Mass flow along the pathways of pharmaceuticals

As listed in Table 2, the median of TE.water for roxithromycin, trimethoprim, ciprofloxacin, cephradine, and cefadroxil are $>20 \%$. These high emission rates suggest a strong need to reduce the emission of these five pharmaceuticals, which may be used as a rationale to prioritize their management. The mass flow studies further showed that the high emission rates resulted from high inflows into NISO and subsequently through to STP. This provides useful information for efficient management, i.e., the focus should be placed on the means to reduce the NISO inflows. However, it should also be noted that no difference in INCN and LEACH resulted among the pharmaceuticals because-due to the lack of information - the supply and the disuse inventory ratios among suppliers and the waste rates of outpatients were assumed to be independent of pharmaceuticals. Once this information becomes available, therefore, the significance of INCN or LEACH could be discriminated in a pharmaceutical-dependent manner. 


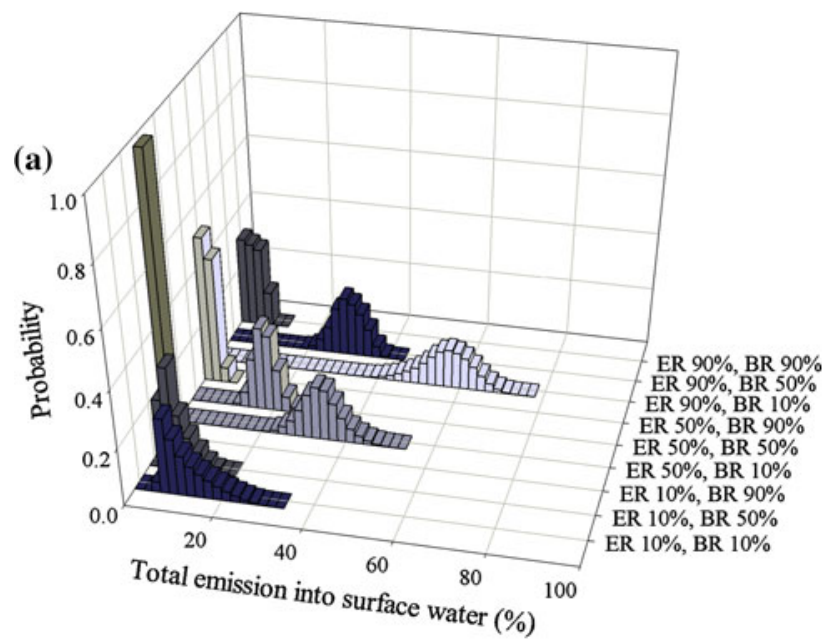

(b)

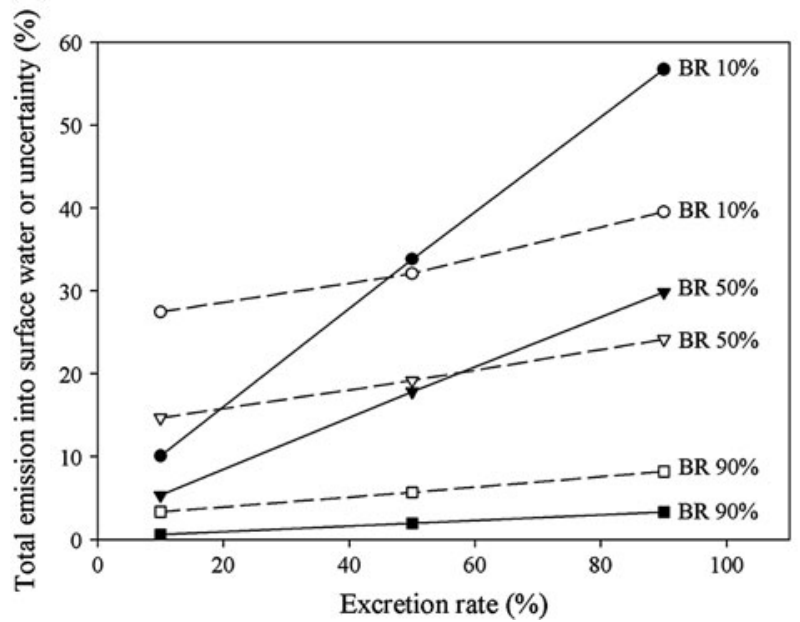

Fig. 5 a Probability distributions of TE.water at various ER and BR.stp, b TE.water or uncertainty of TE.water with respect to ER and BR.stp. Filled symbols TE.water, open symbols and uncertainty. Model parameters are defined in Table 1

\section{Risk characterization and priority setting}

As can be noted in Table 3, the emission ranking and the HQ ranking are not in accordance with each other. As the HQ is a function of two factors, i.e., PEC and toxicity, this discordance could arise from either or both of the two factors. It was noted that the ranking by PEC tends to follow that by emission, indicating that the emission rate dictates the PEC of these 19 pharmaceuticals in water. Therefore, the discordance between the rankings by emission and by HQ should largely be accounted for by the toxicity of the pharmaceuticals. These 19 pharmaceuticals may be divided into three groups from a management perspective. The first group includes pharmaceuticals of high HQ ranking due to high emission (e.g., cimetidine, roxithromycin, and amoxicillin). For this group, the management focus should be placed on emission reduction

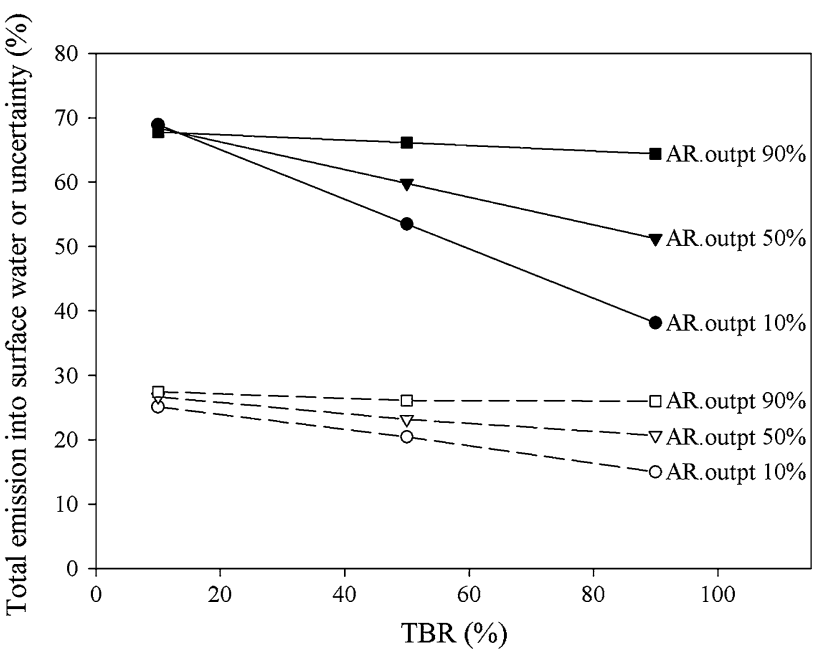

Fig. 6 TE.water or uncertainty of TE.water with respect to TBR. Filled symbols TE.water, open symbols uncertainty. Model parameters are defined in Table 1

measures, such as usage control or Take-back programs The second group is that of high HQ ranking primarily due to high toxicity despite emission not being as high (e.g., acetaminophen, trimethoprim, and erythromycin). The use or development of less or non-toxic alternatives would be a solution if emission is already low. The third is the group of pharmaceuticals of medium to low HQ ranking for which the need of monitoring, as the first step of further management action, should be determined depending on the level of the respective HQ. More details on the management approaches for each of the three groups are presented in ESM 3.

To summarize, we have developed an emission estimation model covering the pathways of pharmaceuticals, including the supply chain, patient administration and personal handling, and various treatment and disposal processes. Based on the uncertainty and sensitivity assessments, we have not only identified the most influencing parameters/variables but have also drawn their management implications. The model estimates, as assessed using PECs, were in agreement with measured values with a disparity less than one order of magnitude. We have demonstrated that the model may potentially be used for the purposes of estimating the emission rates to surface waters and identifying factors critical to reducing these emission rates, as well as be applied to the screening and priority setting of pharmaceuticals.

Acknowledgments This study was funded by KEITI, NRF, and KEI under research grants with contract numbers 412-111-003, 2011-0016767, and 2013-063, respectively.

Conflict of interest None. 


\section{References}

1. Heberer T, Stan HJ. Determination of clofibric acid and $\mathrm{N}$-(phenylsufonyl)-sarcosine in sewage, river and drinking water. Int J Environ Anal Chem. 1997;67(1-4):113-24.

2. Buser HR, Müller MD, Theobald N. Occurrence of the pharmaceutical drug clofibric acid and the herbicide mecoprop in various Swiss lakes and in the North Sea. Environ Sci Technol. 1998;32(1):188-92.

3. Buser HR, Poiger T, Müller MD. Occurrence and fate of the pharmaceutical drug diclofenac in surface waters: Rapid photodegradation in a lake. Environ Sci Technol. 1998;32(22): 3449-56.

4. Daughton CG. Chemicals from the practice of healthcare: challenges and unknowns posed by residues in the environment. Environ Toxicol Chem. 2009;28(12):2490-4.

5. Rodriguez-Mozaz S, Weinberg HS. Meeting report: pharmaceuticals in water-an interdisciplinary approach to a public health challenge. Environ Health Perspect. 2010;118(7):1016-20.

6. Kim JW, Jang HS, Kim JG, Ishbashi H, Hirano M, Nasu K, et al. Occurrence of pharmaceutical and personal care products (PPCPs) in surface water from Mankyung River, South Korea. J Health Sci. 2009;55(2):249-58.

7. Choi K, Kim Y, Park J, Park CK, Kim M, Kim HS, et al. Seasonal variations of several pharmaceutical residues in surface water and sewage treatment plants of Han River, Korea. Sci Total Environ. 2008;405(1-3):120-8

8. Choi K, Kim Y, Jung J, Kim MH, Kim CS, Kim NH, et al. Occurrences and ecological risks of roxithromycin, trimethoprim, and chloramphenicol in the Han river, Korea. Environ Toxicol Chem. 2008;27(3):711-9.

9. National Institute of Environmental Research, Korea (NIER). Development of analytical method and study of exposure of pharmaceuticals and personal care products in environment. 2006 (in Korean). NIER, Incheon.

10. National Institute of Environmental Research, Korea (NIER). Development of analytical method and study of exposure of pharmaceuticals and personal care products in environment (II). 2007 (in Korean). NIER, Incheon.

11. National Institute of Environmental Research, Korea (NIER). Environmental risk assessment of residual pharmaceuticals (I). 2008 (in Korean). NIER, Incheon.

12. National Institute of Environmental Research, Korea (NIER). Environmental risk assessment of residual pharmaceuticals (II). 2009 (in Korean). NIER, Incheon.

13. National Institute of Environmental Research, Korea (NIER). Environmental risk assessment of residual pharmaceuticals (III). 2010 (in Korean). NIER, Incheon.

14. Behera SK, Kim HW, Oh JE, Park HS. Occurrence and removal of antibiotics, hormones and several other pharmaceuticals in wastewater treatment plants of the largest industrial city of Korea. Sci Total Environ. 2011;409(20):4351-60.
15. U.S. Food and Drug Administration (FDA). Guidance for industry-environmental assessment of human drug and biologics applications. 1998. FDA, Washington D.C.

16. European Agency for the Evaluation of Medical Products. Guideline on the environmental risk assessment of medicinal products for human use. 2006. European Agency for the Evaluation of Medical Products, London.

17. Halling-Sørensen B, Nielsen SN, Lanzky PF, Ingerslev F, Lützhøft $\mathrm{HCH}$, Jørgensen SE. Occurrence, fate and effects of pharmaceutical substances in the environment-a review. Chemosphere. 1998;36(2):357-93.

18. Koehler A, Wildbolz C. Comparing the environmental footprints of home-care and personal-hygiene products: the relevance of different life-cycle phases. Environ Sci Technol. 2009;43(22): 8643-51.

19. Daughton CG, Ruhoy IS. Environmental footprint of pharmaceuticals: the significance of factors beyond direct excretion to sewers. Environ Toxicol Chem. 2009;28(12):2495-521.

20. National Institute of Environmental Research, Korea Republic. Development of analytical method and study of exposure of residual pharmaceuticals (II). 2009 (in Korean). NIER, Incheon.

21. Korea Pharmaceutical Manufacturers Association (KPMA). Pharmaceutical production in 2009. 2010 (in Korean). KPMA, Seoul.

22. Korea Pharmaceutical Information Center. Monograph. Available at: http://www.health.kr. Accessed 4 June 2012.

23. Druginfo. Monograph. Available at: http://www.druginfo.co.kr. Accessed 4 June 2012.

24. American Society of Health-System Pharmacists. AHFS DI monographs. Available at http://www.drugs.com/monograph. Accessed 4 June 2012.

25. Gentry B. Oracle crystal ball user manual, release 11.1.1.1.00. Oracle; 2008.

26. Korea Zero Waste Movement Network (KWMN). Proper usage of domestic pharmaceuticals and pilot project of pharmaceutical Take-Back program. 2009 (in Korean). KWMN, Seoul.

27. Korea Zero Waste Movement Network (KWMN). Proper usage of domestic pharmaceuticals and pilot project of pharmaceutical Take-Back program. 2010 (in Korean). KWMN, Seoul.

28. Korea Pharmaceutical Manufacturers Association (KPMA). Statistics of Pharmaceutical Industry. 2010 (in Korean). KPMA, Seoul.

29. Ministry of Environment, Korea. 2009 Statistics of sewerage. 2010 (in Korean). Ministry of Environment, Seoul.

30. Korea Zero Waste Movement Network (KWMN). Treatment method of pharmaceuticals disposed by pharmaceutical-using institutes. 2009 (in Korean). KWMN, Seoul. Available at: http:// www.waste21.or.kr.

31. Cleuvers M. Mixture toxicity of the anti-inflammatory drugs diclofenac, ibuprofen, naproxen, and acetylsalicylic acid. Ecotoxicol Environ Saf. 2004;59(3):309-15. 